\title{
A two staged treatment procedure for the difficult to treat bladder neck contractures with concomitant incontinence. In the search of a solution to a complex problem
}

\author{
Ioannis Adamakis, Evangelos Fragkiadis, Ioannis Katafigiotis, Giorgos Kousournas, \\ Konstantinos Stravodimos, Constantinos A. Constantinides \\ $1^{\text {st }}$ University Urology Clinic, Laiko Hospital, Greece.
}

\begin{abstract}
Summary Objective: To examine the efficacy of a two staged treating strategy with the use of a non-permanent urethral ALLIUM ${ }^{\circledR}$ stent for the management of recurrent bladder neck stenosis and subsequently the use of an artificial sphincter AUS800 ${ }^{\circledR}$ by AMS for the management of the incontinence.

Materials and Methods: We progressively identified patients eligible for the study creating a population of cases with recurrent bladder neck stenosis and concomitant incontinence occurring after the last intervention for the stenosis. Efficacy for the treatment of the stenosis was defined as no recurrence both prior and post to the sphincter placement and efficacy for the treatment of the incontinence was defined as continence (0-1pads) after the sphincter placement. Results and Limitations: 14 white males with a mean age of 66.21 , ranging from 59 to 73 years consisted the population of the study. All patients had severe stress incontinence following the last transurethral resection. The efficacy of the treatment of the bladder neck stenosis was 93\% (13/14) while the efficacy for the treatment of the incontinence was $100 \%$. A single patient had a recurrent bladder neck stenosis after the artificial sphincter placement and was treated with transurethral resection using a long pediatric $13 \mathrm{~F}$ resectoscope at 12 months. Our limitations is the absence of a control group and the small number of patients enrolled, with a relatively short time of follow up.

Conclusions: In our series we propose the use of a non-permanent urethral ALLIUM ${ }^{\circledR}$ stent for 6 months in order to control the growth of fibrotic scar tissue, a further 6 months follow up for recurrence, and then placement of an artificial sphincter. The results are very promising both on stabilizing the vesicourethral stenosis, and on patient safety and tolerability.
\end{abstract}

KEY WORDS: Vesicourethral anastomosis stenosis; Bladder neck contracture; Urethral stent.

Submitted 10 March 2015; Accepted 30 April 2015

\section{INTRODUCTION}

Vesicourethral anastomosis stenosis and bladder neck contracture is a deferred complication of prostate surgery, predominantly in radical prostatectomy, that occurs in $0.5 \%$ to $14.6 \%$ of the patients (1). A number of risk factors have been clearly described: age, increased Body Mass
Index, previous prostate surgeries, open surgical technique, postoperative haematuria, urinary leak and urinary retention, external beam radiation and biochemical recurrence $(2,3)$. The vast majority of anastomotic strictures manifest within the first year post operatively (4).

Different management approaches have been proposed according to the severity of each case, such as dilation, laser or cold cut incisions, use of corticosteroids, transurethral resection of fibrotic tissue, revision surgery and urinary diversion surgery. No consensus has been reached in their management and, despite the use of different and combined techniques, the need for repeat treatment remains as high as $70 \%$ depending on the method (5). In the more severe and relapsing cases, patients end up incontinent after multiple treatments, while obstruction is refractory to usual endoscopic modalities. It is evident that bladder neck contractures cause seriously morbidity and affect the quality of life of patients while the cost rises due to multiple procedures. We present the results of our prospective study of a new treatment modality for patients with severe symptoms caused by bladder neck obstruction, recalcitrant to multiple and different endoscopy treatment modalities. All patients had developed severe stress incontinence after previous endoscopic treatments for bladder neck obstruction. Patients were treated with the placement of a non-permanent urethral ALLIUM $^{\circledR}$ stent (6) for 6 months in order to control the growth of fibrotic scar tissue. After that the stent was removed and the patients were further followed up for 6 months and if remained unobstructed an artificial sphincter was placed to treat incontinence.

\section{MATERIALS AND METHOdS}

Patient characteristics are depicted in Table 1. Fourteen white males with a mean age of 66.21 , ranging from 59 to 73 years consisted the population of the study. Ten patients were subjected to open radical prostatectomy as primary surgery, 1 patient to radical cystectomy and orthotopic neobladder, 2 patients to open transvesical adenomectomy and 1 patient to transurethral prostatectomy.

Patient inclusion criteria were recurrent bladder neck 
Table 1.

Patient's clinical details.

\begin{tabular}{|lccll}
\hline \begin{tabular}{l} 
Patient characteristics \\
\hline Number of patients
\end{tabular} & 14 patients & & & \\
\hline Patient age & 66,21 & $59-73$ age range & & \\
\hline Primary surgery & 10 open RRP & 1 open R-C & 2 open TVA & 1TUR-P \\
\hline $\begin{array}{l}\text { Number of transurethral resections } \\
\text { prior to trial enrollment }\end{array}$ & 3,64 & $2-7$ & \\
\hline Patients with other treatments applied & 3 laser incisions & 3 application of corticosteroids & \\
\hline Severe incontinence & 14 patients & & \\
\hline PSA prior to enrollment & $<0,01$ & & \\
\hline
\end{tabular}

Table 2.

Results.

\begin{tabular}{|lcc|}
\hline Results & \multicolumn{1}{l}{} \\
\hline Days after resection for stent placement & 6,25 & $6-7$ \\
\hline Months of indwelling stent & 6,14 & $6-6,5$ \\
\hline Follow up after stent removal and time of sphincter placement & $0 / 14$ & \\
\hline Bladder neck stenosis at sphincter placement & 12,93 & $11-16$ \\
\hline Follow up after sphincter placement & $14 / 14$ & \\
\hline Continence achieved (0-1 pad per day) & $1 / 14$ & Self reported at 12 months \\
\hline Recurrence of bladder neck stenosis & & \\
\hline Transurethral resection & & \\
\hline
\end{tabular}

Table 3.

Complications.

\begin{tabular}{|lccc|}
\hline Complications & No of patients & Intervention & Clavien score \\
\hline Stent migration & $1 / 14$ after 1 month & Replacement & 3,1 \\
\hline Acute urinary retention & $1 / 14$ immediately & Suprapubic urine drainage \& NSAID & 3,1 \\
\hline Perineal pain & $2 / 14$ & Paracetamol & 1 \\
\hline Dysuria & $8 / 14$ & Self resolved & 1 \\
\hline
\end{tabular}

sedation and fluorescence control for proper placement and stent measurement after 1 week. Figure 1. The stent was removed after 6 months, and patients were followed up for stenosis recurrence at 1,3 and 6 months with post voiding residual ultrasound and at 6 months with urethrography. If patients were unobstructed at 6 months, an artificial sphincter AUS800 ${ }^{\circledR}$ by AMS was placed with open perineal surgery and patients were again followed up at 1 , 3, 6 and 12 months (mean follow up time 12.93 months). Treatment scheme is reported in Figure 2.

The ALLIUM ${ }^{\circledR}$ stent is a $45 \mathrm{~F}$ triangular polymer coated stent designed for high flexibility, low irritation, long indwelling time, reduced migration due to transsphincter anchor, acting as a wide mold to the development of scar tissue and allowing for time of fibrotic tissue to stabilise. ALLIUM ${ }^{\circledR}$ stent is provided in $45 \mathrm{~mm}$ and $55 \mathrm{~mm}$ length. obstructions after at least 2 transurethral resections of fibrotic tissue post primary surgery, as well as multiple treatment modalities such as laser incision and use of corticosteroids. All patients had severe stress incontinence following the last transurethral resection.

In all patients with a history of prostate and bladder cancer, a relapse was excluded through digital rectum examination (DRE), prostatic specific antigen (PSA) and pathology report of the transurethral resection. The clinical study was approved by the scientific committee of our hospital and all the patients signed a written consent after a detailed presentation of our treatment approach The primary objective was to examine the efficacy in the treatment of the recurrent urethrovesical contracture after the placement of the sphincter in the 12 month follow up.

The secondary objective was to examine the efficacy of the treatment of the severe stress incontinence induced over multiple recurrences and treatments after the placement of the sphincter in the 12 month follow-up.. All complications were recorded after patient evaluation and clinical examination and classified according to the Clavien-Dindo Score (Table 3).

Patients were treated by a single surgeon with an aggressive transurethral resection of obstructive fibrotic tissue. Placement of the ALLIUM ${ }^{\circledR}$ stent was facilitated under

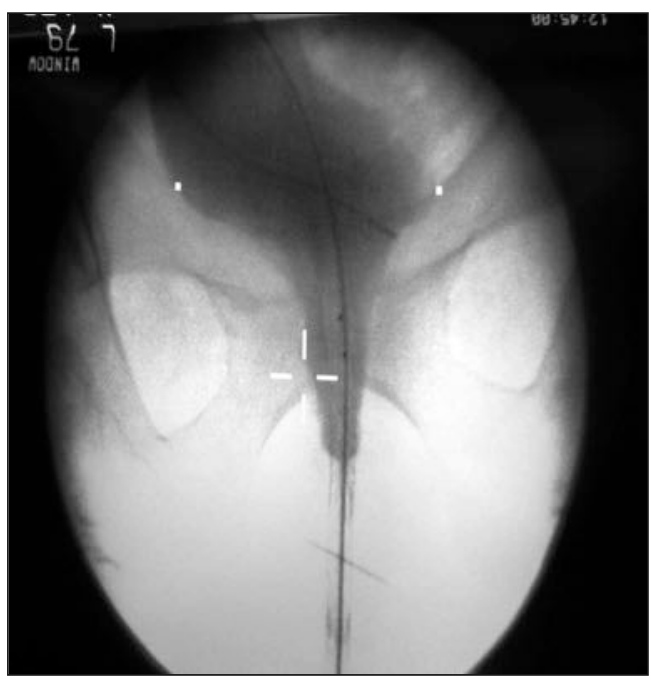

Figure 1.

Stent

Placement.

\section{RESULTS}

All 14 patients remained unobstructed for the complete time of follow up prior to sphincter placement, after the removal of the ALLIUM ${ }^{\circledR}$ stent. A single patient has relapsed after the artificial sphincter placement and was treated with transurethral resection using a long pediatric $13 \mathrm{~F}$ resectoscope at 12 months and as a result the 
Figure 2.

Treatment scheme.

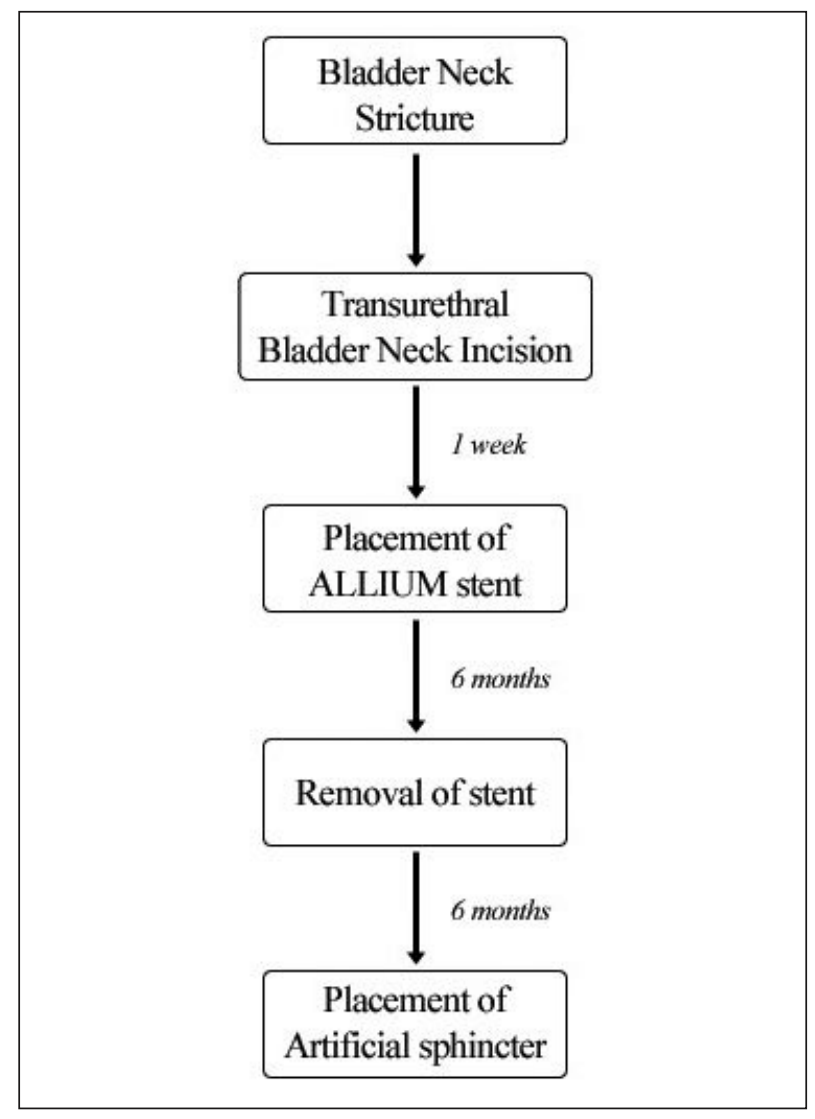

final efficacy was 93\%. All 14 patients regained full continence after the application of the sphincter using 0-1 light pad per day (Efficacy 100\%). Patients results are reported in Table 2 . In 1 patient, acute urinary retention occurred immediately after the stent placement, resolved by the use of suprapubic urine drainage and NSAID's for one week, then suprapubic drainage was removed and patient was unobstructed. One case of stent migration at 1 month was reported with successful replacement of the stent. The stent was well tolerated with mild dysuria complaints in 8 cases, 2 patients further reported perineal pain, well controlled with paracetamol. All patients had no dysuria or pain after stent removal.

Complications are reported in Table 3.

\section{Discussion}

Treatment of vesicourethral anastomosis stenosis is very challenging, due to the heterogeneity of the patients requiring different therapeutic approaches. Furthermore the high relapse rates affect significantly patient's quality of life adding both to the morbidity and the cost due to the demand for multiple operations.

For cases with minimal fibrotic tissue the initial approach usually is the transurethral dilating balloon with success rates of $59 \%$, but with recurrence rates of $70 \%$ with the occurrence of dense scar tissue as the cause of treatment failures (5). Use of cold knife incision has also been suggested as the initial approach of strictures in continent patients, with good continence clinical results similar to patients with no strictures after radical prostatectomy in a 18.9 months follow up (7). Giannarini et al. investigated the efficacy of balloon dilation compared to multiple cold cut incision over an extended follow up of 48 months, establishing the superiority of the cold cut approach, but also the frequent need of re-treatment (26\%), the preservation of continence in all patients and the improvement of incontinence prior to treatment (4). In an attempt to improve long term results multiple treatment options have been proposed. The cold knife incision has been combined with the in situ injection of mitomycin C, reaching an $89 \%$ success rate, but still requiring multiple treatments (8).

More complicated cases with contractures due to dense fibrotic tissue without sphincter involvement and without stress incontinence are usually treated with the facilitation of cautery incision and resection or laser. The use of the transurethral resection of bladder neck contractures has been proven safe when the obstruction was located below the bladder neck musculature, well above the distal urethral sphincter (9). Comparing the resection of the stricture with a bipolar endoscopic resection and Holmium laser treatment similar success results has been reported, but more durable in the case of bipolar use, with the same complication rates regarding continence (10). In the same principal, plasma vaporisation has been compared with transurethral resection in bladder neck sclerosis, post monopolar transurethral resection, with bipolar modality showing superior efficacy, a satisfactory safety profile in similar medium-term follow-up (11). Collins knife incision has also been used in neovesical strictures over transurethral resection, but with the assistance of continuous intermittent catheterisation to preserve the results (12). The previous studies treated patients with mild obstruction and with none or mild to moderate incontinence, hence different than our group,which consisted of patients with severe obstruction and severe incontinence.

An even more difficult to treat subset of patients have bladder neck contractures that involve the sphincter causing stress incontinence, that are due to previous endoscopic treatments affecting the sphincter so adding incontinence to the symptoms along with recurrence. A bladder neck incision technique with a Collins knife electrocautery has been used for the treatment of these more challenging cases, as part of a two staged procedure dealing with the bladder neck contracture first, and at a deferred stage with the incontinence, but still with a high recurrence rate of 25\% (13). The use of Holmium laser incision with the adjuvant use of triamcinolone has been proposed for incontinent patients relapsing after previous treatment, with placement of the artificial sphincter as early as 2 months, but with a high retreatment rate of almost 29\% (14)

In patients refractory to endoscopic approach, more invasive solutions such as perineal reconstructive surgery or placement of urethral stents has been suggested (15). In similar populations with complicated strictures or contractures, but with concomitant incontinence, a two staged management of open reconstructive surgery and artificial sphincter placement seems to be the primary 
choice (16). In order to avoid the copious reconstructive perineal surgery Magera et al. opted for multiple stent placement and implantation of an artificial sphincter, achieving success rates of $76 \%$ (17). Elliot et al. with the same approach and the use of a permanent stent, reported a success rate $89 \%$ with mild pain symptoms (18). This more complex patient subset with a history of multiple endoscopic treatments, high recurrence rate and severe stress incontinence, trapped in a vicious cycle of incontinence to retention and back, is closer to our case series.

Breyer et al. in an excellent review on the management of refractory bladder neck contractures after radical prostatectomy, summarised the shortcomings of stents (19). Stents, being a foreign body, carry a lifelong infection risk. Stent placement can induce urinary urgency, frequency or dysuria if it is near the trigone, and if it is placed too distally, perineal discomfort may occur in a sitting position. A restenosis of the stent may occur, due to either recurrent scar tissue or to calculi, requiring endoscopic excision. When stenosis recurs after the placement of the artificial sphincter the repeated endoscopic interventions may harm the artificial urinary sphincter (AUS). Finally, once placed, the stent is extremely difficult to remove, requiring an open surgical approach. Most of these disadvantages are intrinsic to the permanent nature of stents, thus using a non permanent stent such as ALLIUM ${ }^{\circledR}$ stent we possibly can circumvent them.

The two stage treatment seems to be well established in patients with severe anastomosis strictures and severe incontinence. The primary step of this two stage treatment is to establish a stable, open vesicourethral anastomosis and, in most cases, specialised perineal reconstructive surgery is being utilised. The use of permanent stents to replace complex open surgery is an intriguing possibility, with proven similar efficacy to reconstructive surgery. On the other hand the utilisation of a permanent stent has some disadvantages, inherent to the use of a foreign body including urgency, frequency, dysuria, pain, infection, and in some cases restenosis. The two stage treatment always facilitates placement of an artificial sphincter to successfully treat incontinence.

In our series we propose the use of a non-permanent urethral ALLIUM ${ }^{\circledR}$ stent for 6 months in order to control the growth of fibrotic scar tissue, and, after a further 6 months follow up for recurrence, placement of an artificial sphincter. The results are very promising in order to stabilize the vesicourethral stenosis with patient safety and tolerability and the procedure is easy to apply in a standard facility setting. Most important is the fact that no stent is left indwelling after the first 6 months, thus bypassing most of the disadvantages mentioned in relation to permanent stents (i.e. risk of infection, pain, discomfort, difficulty of endoscopic procedures and removal) without compromising recurrent stenosis or successful long lasting results. As our series demonstrate, all 14 patients remained stable and unobstructed in the 6 month follow up period and the single recurrence 9 months after sphincter placement was readily resolved with a transurethral resection, thus 13/14 patients were recurrence free for the complete follow up period. All 14 patients regained continence after the application of the artificial sphincter.

Our limitations is the absence of a control group and the small number of patients enrolled, with a relatively short time of follow up. Although this is an initial case series study, of a very complicated difficult to treat group of patients, with extended stenosis, multiple recurrence and severe incontinence, the follow up scheme is strict and comparable to similar studies. Due to the solid promising results of our novel treatment modality it should be considered as a candidate to add to the armament of the urologist in treating complex cases of vesicourethral contractures. A comparative randomised clinical trial with a larger patient sample and longer follow up is definitely needed to establish the right method for the right patient.

\section{REFERENCES}

1. Heidenreich A, Bastian PJ, Bellmunt J, et al. EAU guidelines on prostate cancer. 2013;160:310.

2. Wang R, Wood DP Jr, Hollenbeck BK, et al. Risk factors and quality of life for post-prostatectomy vesicourethral anastomotic stenoses. Urology. 2012; 79:449-57

3. Sandhu JS, Gotto GT, Herran LA, et al. Age, obesity, medical comorbidities and surgical technique are predictive of symptomatic anastomotic strictures after contemporary radical prostatectomy. J Urol. 2011; 185:2148-52.

4. Giannarini G, Manassero F, Mogorovich A, et al. Cold-knife incision of anastomotic strictures after radical retropubic prostatectomy with bladder neck preservation: efficacy and impact on urinary continence status Eur Urol. 2008; 54:647-656.

5. Ramchandani P, Banner MP, Berlin JW, et al. Vesicourethral anastomotic strictures after radical prostatectomy: efficacy of transurethral balloon dilation. Radiology 1994; 193:345-349.

6. Yachia D, Markovic Z, Markovic B, Stojanovic V. Endourethral prostheses for urethral stricture. Acta Chir Jugosl. 2007; 54:105-14.

7. Yurkanin JP, Dalkin BL, Cui H. Evaluation of cold knife urethrotomy for the treatment of anastomotic stricture after radical retropubic prostatectomy J Urol. 2001; 165:1545-1548.

8. Vanni AJ, Zinman LN, Buckley JC. Radial urethrotomy and intralesional mitomycin $C$ for the management of recurrent bladder neck contractures. J Urol. 2011; 186:156-60.

9. Popken G, Sommerkamp H, Schultze-Seemann W, et al. Anastomotic stricture after radical prostatectomy. Incidence, findings and treatment. Eur Urol. 1998; 33:382-6.

10. Brodak M, Kosina J, Pacovsky J, et al. Bipolar transurethral resection of anastomotic strictures after radical prostatectomy. $J$ Endourol. 2010; 24:1477-81.

11. Geavlete B, Moldoveanu C, Iacoboaie C, Geavlete P. Plasma vaporization versus standard transurethral resection in secondary bladder neck sclerosis: a prospective, medium-term, randomized comparison. Ther Adv Urol. 2013; 5:75-83.

12. Patel SG, Cookson MS, Clark PE, et al. Neovesical-urethral anastomotic stricture after orthotopic urinary diversion: presentation and management. BJU Int. 2008; 101:219-22.

13. Gousse AE, Tunuguntla HS, Leboeuf L. Two-stage management of severe postprostatectomy bladder neck contracture associated with stress incontinence. Urology. 2005; 65:316-9. 
14. Eltahawy E, Gur U, Virasoro R, et al. Management of recurrent anastomotic stenosis following radical prostatectomy using holmium laser and steroid injection. BJU Int. 2008; 102:796-8.

15. Elliott SP, McAninch JW, Chi T, et al Management of severe urethral complications of prostate cancer therapy. J Urol. 2006; 176:2508-13.

16. Simonato A, Gregori A, Lissiani A, Carmignani G. Two-stage transperineal management of posterior urethral strictures or bladder neck contractures associated with urinary incontinence after prostate surgery and endoscopic treatment failures. Eur Urol. 2007; 52:1499-504
17. Magera JS Jr, Inman BA, Elliott DS. Outcome analysis of urethral wall stent insertion with artificial urinary sphincter placement for severe recurrent bladder neck contracture following radical prostatectomy. J Urol. 2009; 181:1236-41.

18. Elliott DS, Boone TB. Combined stent and artificial urinary sphincter for management of severe recurrent bladder neck contracture and stress incontinence after prostatectomy: a long-term evaluation. J Urol. 2001; 165:413-5.

19. Breyer BN, McAninch JW. Management of recalcitrant bladder neck contracture after radical prostatectomy for prostate cancer. Endoscopic and open surgery. J Urol. 2011; 185:390-1.

\section{Correspondence}

Ioannis Adamakis, MD Assistant Professor

yianton@hotmail.com

Evangelos Fragkiadis, MD Urologist - Scientific Associate

e.fragkiadis@gmail.com

Giorgos Kousournas, MD Resident

giorgoskousournas@gmail.com

Konstantinos Stravodimos, MD Associate Professor

kgstravod@yahoo.com

Constantinos A. Constantinides, MD Professor of Urology

ckonstan@med.uoa.gr

Ioannis Katafigiotis MD, MLS, PhD (Corresponding Author) Resident katafigiotis@yahoo.com

Department of Urology, Athens University Medical School,Greece

Laiko Hospital

17 Agiou Thoma str., 11527, Athens, Greece 